\title{
Effect of berberine on the HPA-axis pathway and skeletal muscle GLUT4 in type 2 diabetes mellitus
} rats

This article was published in the following Dove Press journal: Diabetes, Metabolic Syndrome and Obesity: Targets and Therapy

\author{
Jia $M i^{1, *}$ \\ Wenda $\mathrm{He}^{1, *}$ \\ Jiawei $L v^{\prime}$ \\ Kai Zhuang' \\ Heqing Huang ${ }^{1,2}$ \\ Shijian Quan' \\ 'School of Pharmaceutical Sciences, \\ Guangzhou University of Chinese \\ Medicine, Guangzhou, People's Republic \\ of China; ${ }^{2}$ Laboratory of Pharmacology \& \\ Toxicology, School of Pharmaceutical \\ Sciences, Sun Yat-sen University, \\ Guangzhou, People's Republic of China \\ *These authors contributed equally to \\ this work
}

Purpose: Activation of the hypothalamus-pituitary-adrenal (HPA) axis pathway is closely related to insulin resistance (IR), glucose, and lipid metabolism disorders in type 2 diabetes mellitus (T2DM). Berberine (BBR) has effect on regulating disorder of glucose and lipid metabolism in T2DM. In fact, activation of the HPA axis pathway is closely related to IR, glucose, and lipid metabolism disorders in T2DM. Here, we investigated whether the therapeutic effect of BBR on T2DM rats is acted through the HPA axis pathway.

Methods: In this research, we investigated the effects of BBR on the HPA-axis pathwayrelated indicators and expression of skeletal muscle glucose transporter 4 (GLUT4) in the high-fat diet and streptozotocin-induced T2DM rats, and identify its possible mechanism of improving IR in T2DM.

Results: BBR significantly reduced fasting blood glucose, total cholesterol, and low-density lipoprotein cholesterol in model rats. It also improved the abnormalities of the high-density lipoprotein cholesterol, the insulin resistance index, the insulin sensitivity index, glucagon, and insulin levels. BBR decreased levels of hypothalamic Orexin-A, the OX2R receptor, the corticotropin-releasing hormone, the pituitary and the plasma adrenocorticotropic hormone, as well as serum and urine corticosterone. At the same time, BBR increased mRNA and protein expressions of GLUT4 in skeletal muscles of model rats as well.

Conclusion: Those results suggested that BBR can exert inhibition on the HPA-axis and increased skeletal muscle expression of GLUT4 proteins, which may be one of the important mechanisms in BBR to improve IR and regulating glucose and lipid metabolism in T2DM rats.

Keywords: berberine, type 2 diabetes mellitus, insulin resistance, the HPA-axis, GLUT4

\section{Introduction}

Type 2 Diabetes Mellitus (T2DM) is one slowly progressing metabolic disease characterized by elevated blood glucose, and the incidence has kept on rising. ${ }^{1}$ Insulin resistance (IR) is an important to base of the pathogenesis of T2DM. ${ }^{2}$ IR leads to the disturbance in the lipid and glucose metabolism, the mechanism of IR is very complicated, ${ }^{3}$ one the mechanism of IR is closely related to the disorder of the hypothalamus-pituitary-adrenal (HPA)-axis.

It was reported that the activity of the HPA-axis is increased in patients with T2DM. Inhibiting the activity of the HPA-axis can improve IR. ${ }^{4,5}$ The HPA-axis is regulated by hypothalamic Orexin-A, which stimulates releasing of corticotropinreleasing hormone $(\mathrm{CRH})$ by activating $\mathrm{OX} 2 \mathrm{R}$ receptor in the lateral hypothalamic
Correspondence: Heqing Huang; Shijian Quan

Department of School of Pharmaceutical Sciences, Guangzhou University of Chinese Medicine, No. 232 East Wai Huan Road, Guangzhou 510006, People's Republic of China

Tel +86 I 39221 I 9719

Email Huangheq@mail.sysu.edu.cn; quansj@gzucm.edu.cn 
region. ${ }^{6} \mathrm{CRH}$ stimulates adrenocorticotropic hormone (ACTH) of pituitary releasing which promotes glucocorticoid (GC) producing and secreting. GC has a wide range of functions in the body and plays a key role in regulating glycolipid metabolism and IR, ${ }^{7}$ which is the most important factor of hyperglycemia, dyslipidemia, and hypertension. ${ }^{8}$ GC can decrease the insulin sensitivity of peripheral tissues and inhibit the translocation of glucose transporter 4 (GLUT4), ${ }^{9}$ which is the important carriers to transport glucose in mammals, present in insulin-sensitive peripheral tissues, mainly skeletal muscles. Glucose does not pass through the cell membrane freely, which needs the assistance of GLUT4, stimulating by insulin signal, GLUT4 translocates to the cell membrane and promotes glucose entry into the cell. ${ }^{10,11}$ When the HPA-axis is in disorder, which affects the translocation of GLUT4 in muscle tissue, reduces the uptake and utilization of glucose, which will directly lead to the occurrence and development of T2DM. ${ }^{12,13}$

Berberine (BBR) is a quaternary ammonium isoquinoline alkaloid, which is found in plants such as the family Berberidaceae, Papaveraceae, Ranunculaceae, Rutaceae, and Menispermaceae. BBR is the primary active ingredient of Chinese medicine Coptis chinensis and Scutellaria baicalensis. Accumulated studies have shown that BBR has a therapeutic effect on $\mathrm{T} 2 \mathrm{DM},{ }^{14}$ which paly an important role in regulating glucose as well as lipid metabolism disorders and improving IR. ${ }^{15-19}$ However, whether it is working through the HPA-axis pathway is yet to be determined.

In this study, we investigated the effects of BBR on the HPA-axis pathway-related indicators and the expression of GLUT4 proteins of skeletal muscle in T2DM rats induced by high-fat and low-dose streptozotocin (STZ), and identified the possible action mechanism of improving IR of BBR.

\section{Materials and methods}

\section{Reagents}

BBR was provided by Weikeqi Biological Technology Co., Ltd. (Sichuan, China). Metformin (MET), carboxymethyl cellulose sodium (CMC-Na), and STZ were purchased from Sigma-Aldrich (California, USA). Bestar ${ }^{\mathrm{TM}}$ qPCR RT kit and Bestar SYBY Green qPCR Master mix were purchased from DBI (Ludwigshafen, Germany). All test assay kits and the CORT, ACTH, CRH, Orenxin A, and OX2R enzyme-linked immunosorbent assay (ELISA) kits were supplied by NanJing JianCheng Bioengineering Institute (Nanjing, China). The full wavelength enzyme marker was provided by Thermo Fisher Scientific Co., Ltd. (USA). The anti-GLUT4 antibody was obtained from Abcam (Cambridge, UK). The Anti-GAPDH rabbit polyclonal antibody and the high sensitivity chemiluminescence detection kit and BCA protein assay kit were purchased from Kangwei Century Biology Co., Ltd. (Beijing, China). Goat anti-Rabbit IgG was provided by Lianke Bio Company (Hangzhou, China). The BBR was dissolved with $0.5 \% \mathrm{CMC}-\mathrm{Na}^{20}$ The MET was dissolved with water.

\section{Animals}

Male Sprague-Dawley rats (130-150 g) were provided by the Experimental Animal Center of Guangzhou University of Chinese Medicine (Guangdong, China). All animals were housed at a room $23 \pm 1^{\circ} \mathrm{C}$ and $55 \pm 5 \%$ relative humidity with a $12 \mathrm{hrs}$ light/12 hrs dark cycle. All experiments were approved by the Institutional Animal Care and Use Committee of Guangzhou University of Chinese Medicine. (License No: SCXK 2013-0034) The animal welfare followed in this study was guideline for ethical review of animal welfare in the People's Republic of China.

\section{Induction of T2DM and drug administration}

In this study, T2DM rats models were replicated by means of the high-fat diet (HFD) plus low-dose STZ injections as reported in the literature. ${ }^{21,22}$ MET is considered to be the first-line drug for the treatment of $\mathrm{T} 2 \mathrm{DM},{ }^{23}$ and the hypoglycemic effect of BBR is similar to that of MET. ${ }^{24}$ Therefore, MET is selected as a positive control drug. After one week of adaptation, the rats were randomly divided into the general diet $(\mathrm{GD}, \mathrm{n}=9)$ and the HFD $(n=36)$ groups. They were fed with normal and HFDs, respectively. The HFD comprised sucrose $20 \%$, lard $15 \%$, cholesterol $1.2 \%$, sodium cholate $0.2 \%$, casein $10 \%$, calcium hydrogen phosphate $0.6 \%$, stone powder $0.4 \%$, premix $0.4 \%$, and basic feed $52.2 \%$. After 4 weeks, all rats were fasted for 14 hrs. The HFD group was intraperitoneally injected with $35 \mathrm{mg} / \mathrm{kg}$ STZ dissolved in the citrate buffer ( $\mathrm{pH}$ 4.3-4.4). Three days later, rats with fasting blood glucose (FBG) levels of 11.1-33.3 mmol/L were randomly split into three groups: the T2DM control group, the BBR treatment group, and 
the MET treatment group. The normal control (NC) group was the GD group that was intraperitoneally injected with an appropriate amount of the citrate buffer without STZ. Rats in the $\mathrm{NC}$ and $\mathrm{T} 2 \mathrm{DM}$ control groups were orally administered with the $0.5 \%$ CMC-Na solution $(50 \mathrm{mg}$ / $\mathrm{kg}$ ). Rats in the BBR treatment group were given BBR $(200 \mathrm{mg} / \mathrm{kg}){ }^{25}$ and the ones in the MET treatment group were given MET $(200 \mathrm{mg} / \mathrm{kg}){ }^{26}$ All rats were treated once a day for 4 weeks.

\section{Biochemical analysis}

At the end of the experiment, fresh arterial blood samples were collected by the use of vacuum blood collection tubes and centrifuged at $3500 \mathrm{rpm}$ for $10 \mathrm{mins}$ to obtain serum samples. The levels of FBG, fasting serum insulin (FINS), TC, high-density lipoprotein cholesterol (HDL-C), and low-density lipoprotein cholesterol (LDL-C) in serum were determined by test assay kits. Insulin sensitivity index (ISI) and insulin resistance index (IRI) were calculated in line with the formulas: $\mathrm{ISI}=-\mathrm{Ln}(\mathrm{FBG} \times \mathrm{FINS})$, and $\mathrm{IRI}=(\mathrm{FINS} \times \mathrm{FBG} / 22.5)$.

\section{Pancreatic histology analysis}

After blood samples were taken, pancreatic tissue was isolated and the expression of insulin and glucagon of pancreatic tissue was detected by immunofluorescence double labeling. Pancreatic tissue sections $(4 \mu \mathrm{m})$ were blocked with $4 \%$ paraformaldehyde for $10 \mathrm{mins}$ and heated with the EDTA antigen recovery buffer for 23 mins. When the sections were cooled to room temperature and blocked with BSA for 30 mins, the immune fluorescent primary antibody glucagon (1:300) and insulin (1:600) were incubated overnight at $4^{\circ} \mathrm{C}$. CY3-labeled and FITC-labeled goat anti-rabbit fluorescent secondary antibodies were added dropwise and incubated for 50 mins at room temperature, and the DAPI stain was added dropwise and incubated at room temperature for $10 \mathrm{mins}$ in the dark. Three pancreas sections of each group were placed under a fluorescence microscope (Nikon, Japan) and imaged $(400 \times)$ : the DAPI UV excitation wavelength was 330-380 $\mathrm{nm}$ and the emission wavelength was 420 $\mathrm{nm}$, with blue light being emitted; the FITC excitation wavelength was 465-495 $\mathrm{nm}$ and the emission wavelength was $555 \mathrm{~nm}$, with green light being emitted; the CY3 excitation wavelength was 510-560 $\mathrm{nm}$ and the emission wavelength was $590 \mathrm{~nm}$, with red light being emitted. The insulin and glucagon fluorescent intensities of the images were measured and the relative expression levels were calculated by Image $\mathrm{J}$ software.

\section{Measurement of urine corticosterone (CORT), serum CORT, and plasma ACTH}

After 4 weeks of oral drug treatment, all rats were fasted overnight and then placed in metabolic cages to gather fresh urine samples. The rats were then anesthetized. The fresh arterial blood samples were collected using the vacuum and EDTA-containing blood collection tubes, which were centrifuged at $3500 \mathrm{rpm}$ for 10 mins to obtain correspondently serum and plasma. The levels of CORT in urine, CORT in serum, and ACTH in plasma were determined by ELISA with full wavelength enzyme marker according to the corresponding instructions of ELISA kits.

\section{Measurement of hypothalamus $\mathrm{CRH}$, Orexin A, OX2R, and pituitary ACTH}

After blood samples were taken, rat hypothalamus and pituitary tissues were collected and homogenized with homogenizer (IKA Company, Germany). After homogenization, the supernatant of the hypothalamus and pituitary tissues was collected in accordance with the procedure offered by the ELISA kits manufacturer. Total protein concentration in the supernatants was separately determined by BCA protein assay kit. The CRH, Orexin A, OX2R concentration of the hypothalamus, and ACTH concentration of the pituitary in the supernatants were detected by relevant ELISA kits. The CRH, Orexin A, OX2R, and ACTH expression levels were the ratio of their respective protein concentration to the total protein concentration.

\section{Determination of the skeletal muscle tissue GLUT4 mRNA expression}

Skeletal muscle tissue of rats was isolated. We extracted Total RNA from the tissue via using the TRIzol reagent. Total RNA samples were quantified spectrophotometrically at 260 and $280 \mathrm{~nm}$, with the OD260/OD280 ratio ranging from 1.8 to 2.0 . The mRNA was transcribed into cDNA on the grounds of instructions of the Bester ${ }^{\mathrm{TM}}$ qPCR RT kit. We performed the polymerase chain reaction (PCR) with the ABI 7500 real-time PCR device (New York, USA) by reference to the protocol of Bester SYBR Green qPCR Master mix. The cycling conditions were as follows: $95^{\circ} \mathrm{C}, 120 \mathrm{~s}$ and 45 cycles of $95^{\circ} \mathrm{C} \& 10 \mathrm{~s}, 60^{\circ} \mathrm{C}$ $\& 34 \mathrm{~s}$, and $72^{\circ} \mathrm{C} \& 30 \mathrm{~s}$. Primers of GLUT4 were AGG 
CCGGGACACTATACCC (forward) and ACTTTCTGTG GGGCGTTGAT (reverse). Primers of GAPDH were TGG ATTTGGACGCATTGGTC (forward) and TTTGCACTG GTACGTGTTGAT (reverse). The relative transcript levels were quantified by the $2^{-\Delta \Delta \mathrm{Ct}}$ value method, and the result was exhibited as the percentage of mRNA of normal.

\section{Determination of the skeletal muscle tissue GLUT4 protein expression}

The skeletal muscle samples were homogenized in the appropriate amount of protease and phosphatase inhibitor, and centrifuged at $12,000 \mathrm{rpm}$ for 10 mins under the temperature of $4^{\circ}$ $\mathrm{C}$, with their supernatants being collected. Protein concentration in the supernatants was scaled by utilizing the BCA protein assay kit, and then supernatant concentration was diluted to 50 $\mu \mathrm{g} / \mu \mathrm{L}$. Protein samples to be tested were heated at $100^{\circ} \mathrm{C}$ for 5 mins after the $4 \times$ Loading buffer was added. Fifty micrograms of the protein samples in each group was separated by $8 \%$ SDS-PAGE gel electrophoresis at $80 \mathrm{~V}$ for $30 \mathrm{mins}$, and then transferred onto the polyvinylidene difluoride (PVDF) membrane at $300 \mathrm{~mA}$ for 70 mins. After transferring, the PVDF membrane was blocked with 5\% BSA at indoor temperature for $2 \mathrm{hrs}$, followed by incubation with the primary antibody anti-GLUT4 (1:1000) and anti-GAPDH (1:1000) at the temperature of $4^{\circ} \mathrm{C}$ overnight. The secondary antibody was then added into the samples at room temperature for $2 \mathrm{hrs}$, and the samples were visualized by Tanno 5200 automatic chemiluminescence image analyzer (Tianneng Technology Co., Ltd. Shanghai, China). The images were analyzed and the relative expression levels were calculated by Image J software.

\section{Statistical analysis}

Data were expressed as the mean \pm standard error of the mean (SEM) and analyzed by the Statistical Package for the
A
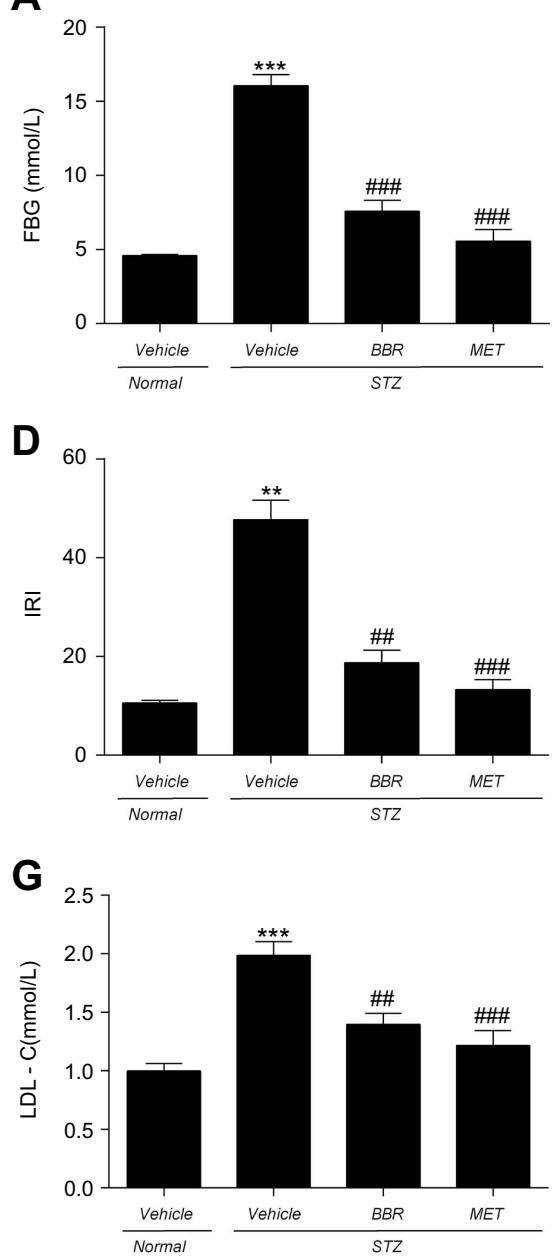

B

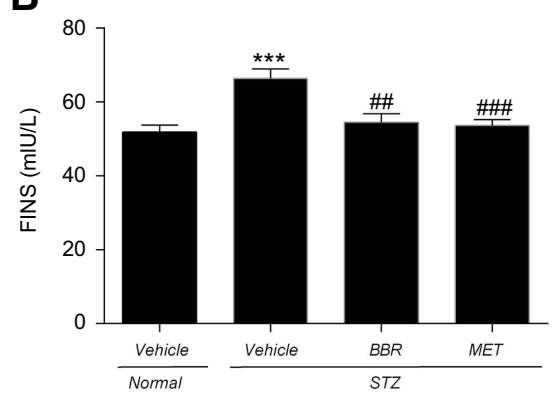

E

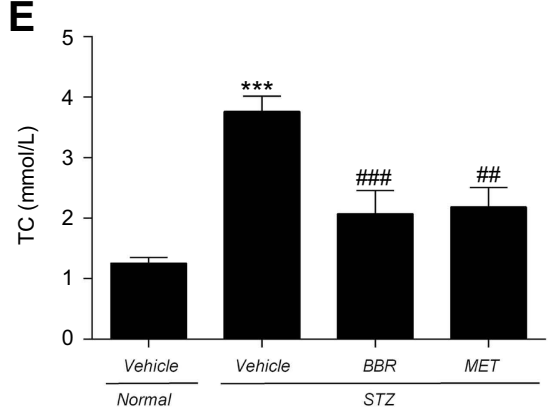

C

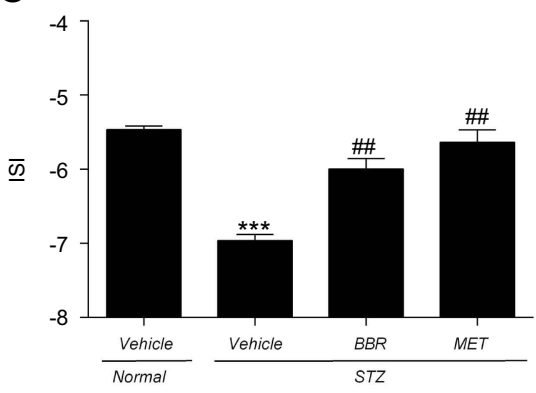

$\mathbf{F}$

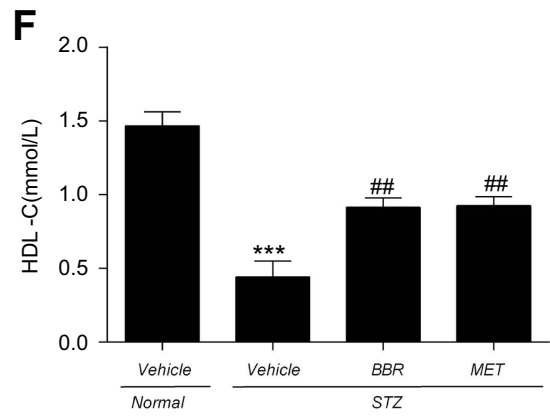

Figure I Effects of BBR on FBG (A), FINS (B), ISI (C), IRI (D), TC (E), HDL-C (F), LDL-C (G) in T2DM rats. Data were expressed as mean \pm SEM $(\mathrm{n}=6)$. ** $p<0.0 \mathrm{I}, * * * p<0.00 \mathrm{I}$ vs. NC group; ${ }^{\#} p<0.01,{ }^{\#} p<0.001$ vs. T2DM control group. 
Social Science version 20.0 (SPSS 20.0). We analyzed remarkable differences of multiple groups by utilizing one-way analysis of variance). $P<0.05$ was considered to be statistically significant.

\section{Results}

Effects of BBR on blood glucose, insulin, and blood lipids in T2DM rats

As shown in Figure 1, the levels of FBG, FINS, IRI, TC, and LDL-C in T2DM control rats were apparently higher than those in the NC group, and the levels of ISI and HDL-C were significantly diminished. BBR markedly decreased the levels of FBG, FINS, IRI, TC, and LDL-C, and increased the levels of ISI and HDL-C when compared with the T2DM control group.

\section{Effects of BBR on insulin and glucagon of pancreas expression in T2DM rats}

Insulin and glucagon secreted by islets in the pancreas were detected by immunofluorescence double labeling. In Figure 2, the nuclei stained by DAPI were blue under ultraviolet excitation, the expression of glucagon was fluorescein-labeled red, and the insulin was green. Insulin and glucagon secretions in T2DM control rats were increased when compared with the

A
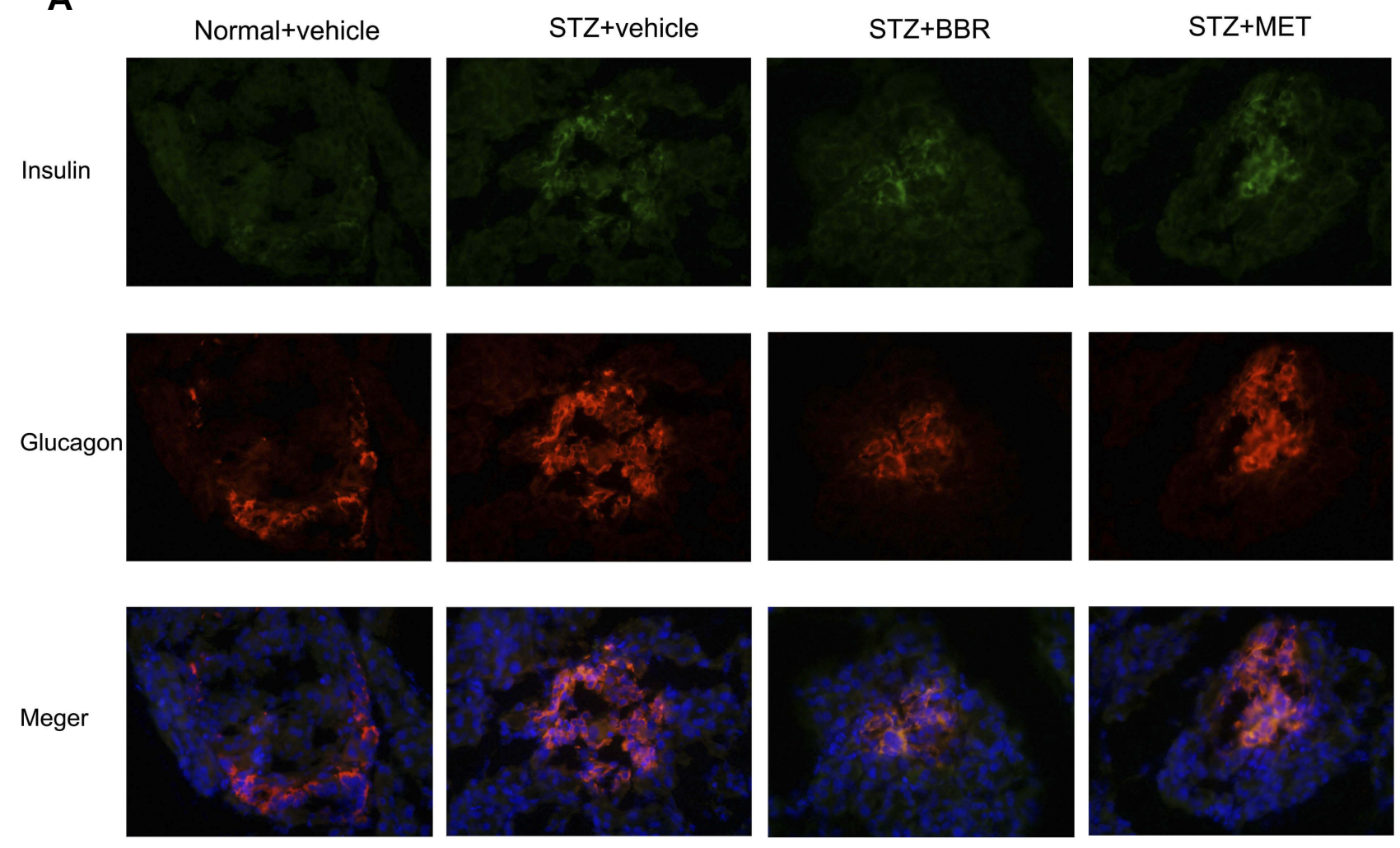

B

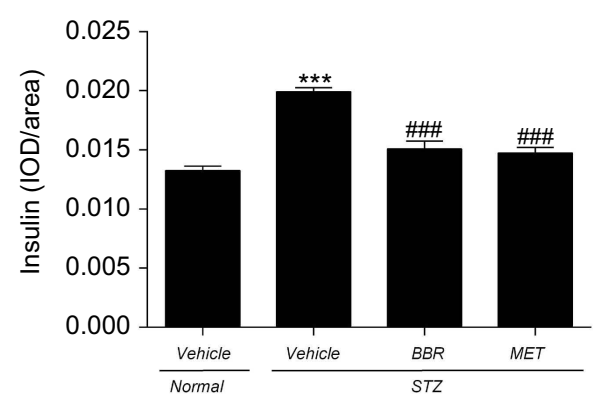

C

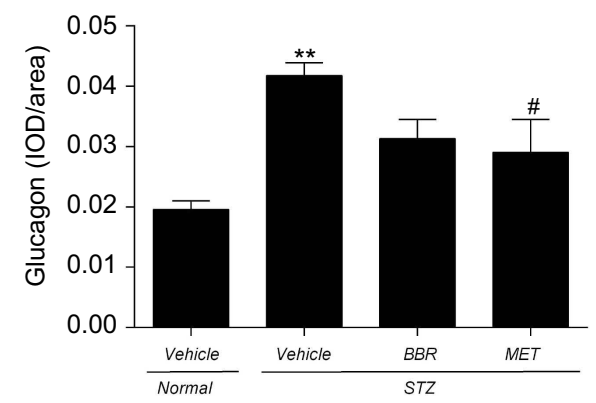

Figure 2 The fluorescent immunogram (A), the expression of insulin (B) and glucagon $(\mathbf{C})$ of pancreasl tissues on T2DM rats between different study groups. Data were expressed as mean \pm SEM $(n=3)$. ${ }^{* *} p<0.001$, ${ }^{* *} p<0.01$ vs. NC group; ${ }^{\# \#} p<0.00$ I, ${ }^{\#} p<0.05$ vs. T2DM control group. 
NC group. However, BBR did decrease insulin levels when compared with the T2DM control group. Although BBR lowered glucagon levels, there was no significant difference compared with the T2DM control group.

\section{Effects of BBR on urine CORT, serum} CORT, and plasma ACTH in T2DM rats

As indicated in Figure 3, urine CORT, serum CORT, and plasma ACTH in T2DM control rats were significantly higher than those in the NC group. BBR significantly reduced the CORT levels in urine and serum when compared with the T2DM control group. In the meanwhile, BBR markedly reduced the plasma ACTH level.

\section{Effects of BBR on hypothalamic Orexin-A, $\mathrm{OX} 2 \mathrm{R}, \mathrm{CRH}$, and pituitary $\mathrm{ACTH}$ in T2DM rats}

As shown in Figure 4, the levels of the hypothalamus Orexin-A, OX2R, CRH, and pituitary ACTH in T2DM control rats were significantly higher than those in the NC group. Compared with the T2DM control group, the BBR treatment group significantly reduced the hypothalamus Orexin-A, OX2R, and the $\mathrm{CRH}$ protein content. ACTH levels in the pituitary also decreased dramatically.

\section{Effect of BBR on skeletal muscle GLUT4 mRNA and protein expression in T2DM rats}

GLUT4 mRNA and protein expression were detected in skeletal muscle tissue. The expression of GLUT4 mRNA and protein on skeletal muscles was decreased in T2DM control rats compared to the NC group. BBR remarkably upregulated GLUT4 mRNA and protein expression when compared with the T2DM control group as shown in Figure 5. However, there was no statistical difference in skeletal muscle GLUT4 mRNA levels in the BBR group compared to the NC group.

B

A
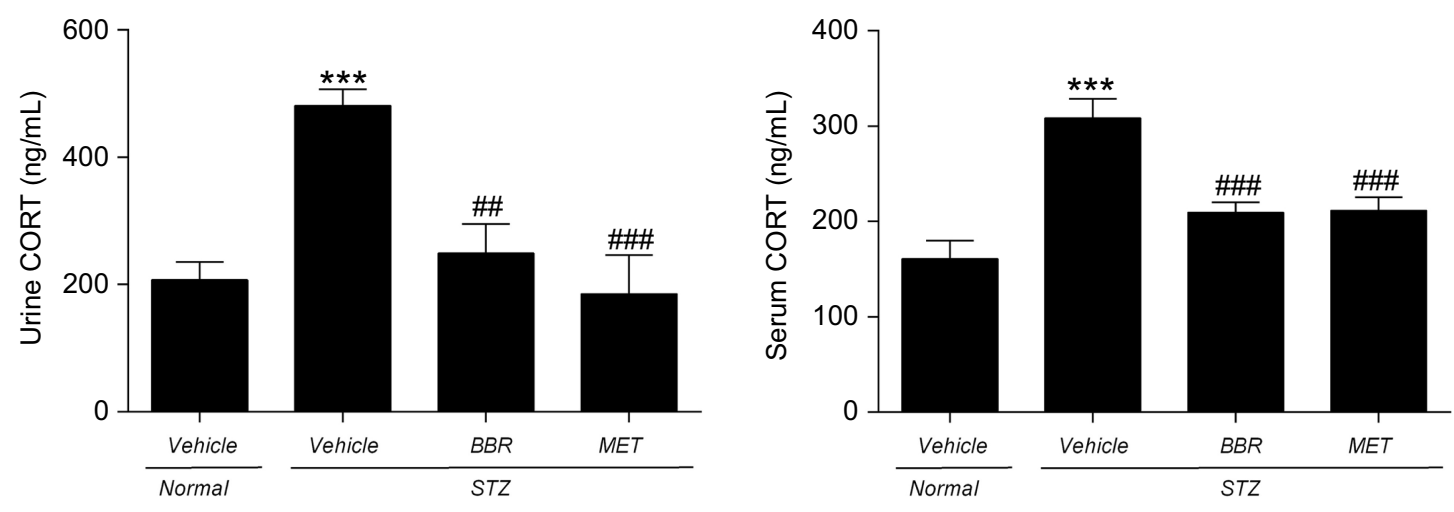

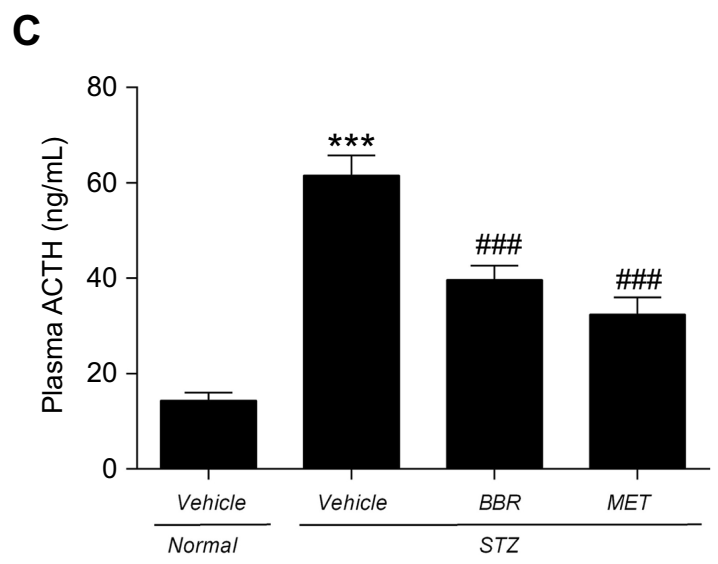

Figure 3 Effects of BBR on urine CORT (A), serum CORT (B), plasma ACTH (C) in T2DM rats. Data were expressed as mean \pm SEM ( $n=6)$. *** $p<0.00$ I vs. NC group; \#<0.0I, ${ }^{\#<0.001}$ vs. T2DM control group. 
A

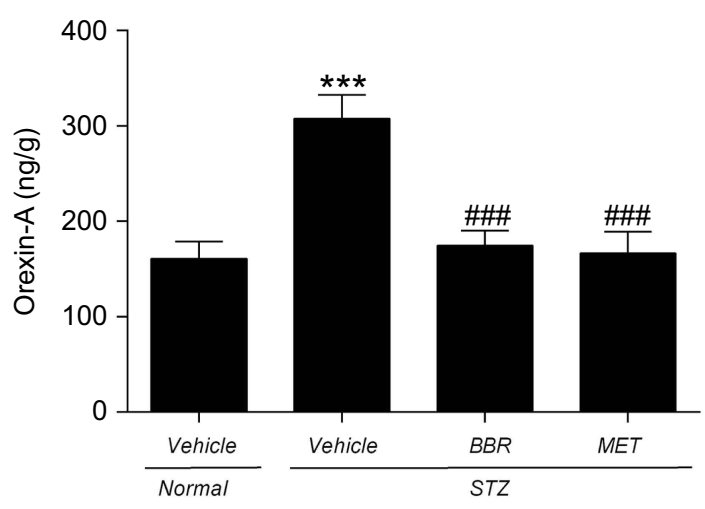

C

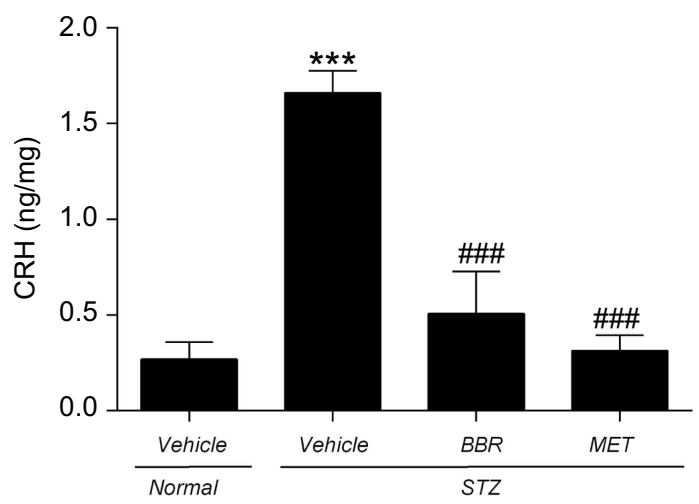

B

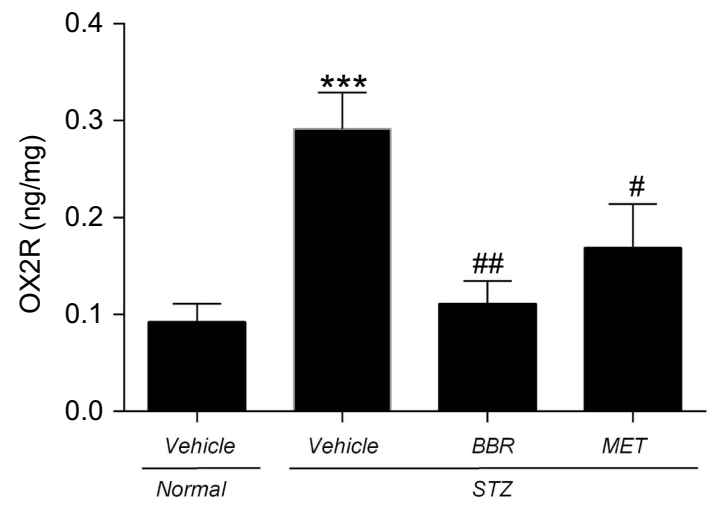

D

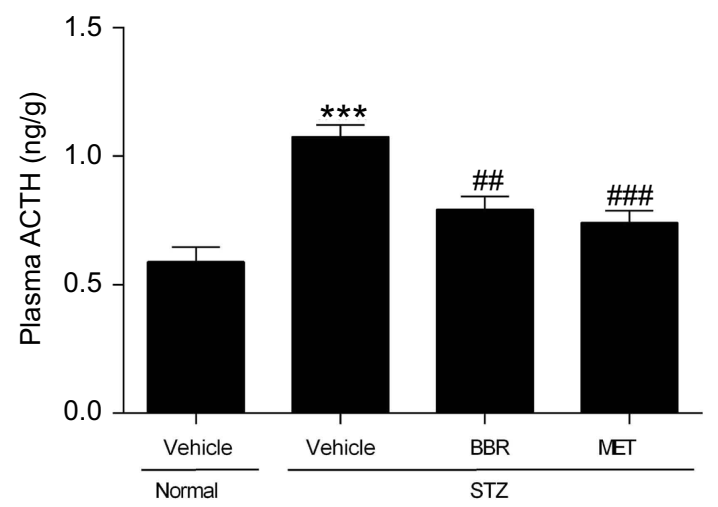

Figure 4 Effects of BBR on hypothalamic Orexin-A (A), OX2R (B), CRH (C) and pituitary ACTH (D) in T2DM rats. Data were expressed as mean \pm SEM ( $n=6)$. *** $p<0.00$ I vs. NC group; ${ }^{\#} p<0.05,{ }^{\#} p<0.01,{ }^{\prime \prime} p<0.001$ vs. T2DM control group.

A

B

GLUT4

GAPDH
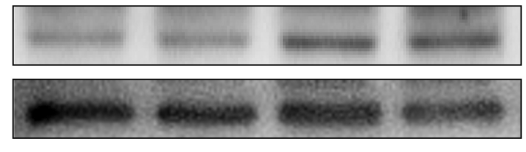

$\begin{array}{cccc}\text { Vehicle } & \text { Vehicle } & \text { MET } & B B R \\ n & & \text { STZ }\end{array}$
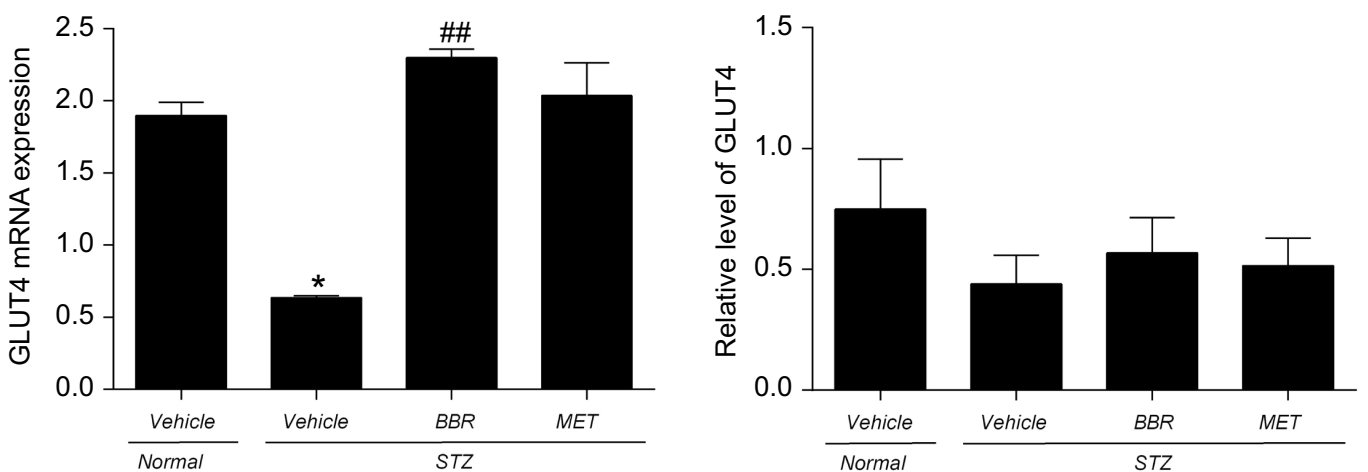

Figure 5 Effect of BBR on skeletal muscle GLUT4 mRNA (A) and protein (B) expression in T2DM rats. Data were expressed as mean \pm SEM $(n=3)$. * $p<0.05$ vs. NC group; $\#_{p<0.01}$ vs. T2DM control group. 


\section{Discussion}

T2DM is the intricate metabolic disorder. As its main pathogenesis, IR is associated with hyperglycemia, dyslipidemia, and effects on insulin sensitivity. ${ }^{2,27}$ In this study, the T2DM model of rats was induced by HFD plus low-dose STZ injection. The results showed that FBG, FINS, IRI, TC, and LDL-C levels were significantly increased in model rats; the levels of ISI and HDL-C were apparently reduced; glucagon and insulin secretion of the islet were increased. They suggested that the T2DM model was successfully replicated (Figures 1 and 2). After the intervention of BBR, the abnormalities of the above indicators were significantly improved. The results confirmed that BBR can improve the glucose and lipid metabolism disorder and IR of T2DM.

Studies indicated that the HPA-axis was hyperactive in people with IR, which was characterized by hypothalamic Orexin A activation of the OX2R receptor releasing $\mathrm{CRH}^{6}{ }^{6}$ pituitary stimulation by $\mathrm{CRH}$, adrenal gland stimulation by ACTH, and increased secretion of CORT. And the negative feedback effect of CORT on the HPA-axis was weakened. $^{28,29}$ CORT was the main effect hormone of the HPA-axis, ${ }^{30}$ which can increase the risk of hyperglycemia and hyperlipidemia to cause IR. ${ }^{4,5,31}$ In this experimental study, the model animals exhibited HPA-axis hyperactivity, which was consistent with the existing findings. Compared with the T2DM control group, BBR significantly inhibited HPA-axis hyperactivity and excessive secretion of CORT (Figures 3 and 4). Those results suggested that BBR could improve the glucose and lipid metabolism disorder and IR of T2DM, which was closely related to its inhibition of the HPA-axis pathway.

GC can reduce the uptake and utilization of peripheral glucose by inhibition of GLUT4 translocation and activity on skeletal muscle. $^{32,33}$ Damage of GLUT4 translocation and activity can affect insulin-stimulated glucose uptake, bringing about IR and hyperglycemia. ${ }^{34,35}$ The better expression and translocation of GLUT4 are beneficial for the improvement of IR in T2DM. ${ }^{36}$ In this experimental study, BBR can increase the expression of GLUT4 mRNA and protein on skeletal muscle of T2DM rats based on inhibition of the HPA-axis pathway (Figure 5), thereby improving IR.

\section{Conclusion}

In summary, this study demonstrates that BBR is efficacious in improving IR in T2DM rats. BBR inhibits HPAaxis hyperactivity in model rats and enhances the GLUT4 gene and protein expression on skeletal muscle. It is preliminarily clarified that BBR inhibition of the HPAaxis pathway is one of the vital mechanisms to improve IR and regulate glucose and lipid metabolism disorder. It provides important information for BBR to improve the neuroendocrine regulation mechanism of IR in T2DM, which deserves further investigation.

\section{Acknowledgment}

This study was financially supported by the Provincial Natural Science Foundation of Guangdong (2017A030313752).

\section{Disclosure}

The authors report no conflicts of interest in this work.

\section{References}

1. Tai N, Wong FS, Wen L. The role of gut microbiota in the development of type 1, type 2 diabetes mellitus and obesity. Rev Endocr Metab Disord. 2015;16(1):55-65. doi:10.1007/s11154-015-9309-0

2. Adeghate E, Saeed Z, D'Souza C, et al. Effect of nociceptin on insulin release in normal and diabetic rat pancreas. Cell Tissue Res. 2018;374(3):517-529. doi:10.1007/s00441-018-2903-1. Epub 2018 August 15.

3. Fazakerley DJ, Krycer JR, Kearney AL, et al. Muscle and adipose tissue insulin resistance: malady without mechanism. J Lipid Res. 2018;jlr.R087510. doi:10.1194/jlr.R087510

4. Daimon M, Kamba A, Murakami H, et al. Association between pituitary-adrenal axis dominance over the renin-angiotensin-aldosterone system and hypertension. J Clin Endocrinol Metab. 2016;101 (3):889-897. doi:10.1210/jc.2015-3568

5. Perry RJ, Zhang XM, Zhang D, et al. Leptin reverses diabetes by suppression of the hypothalamic-pituitary-adrenal axis. Nat Med. 2014;20(7):759-763. doi:10.1038/nm.3579

6. Xu TR, Yang Y, Ward R, et al. Orexin receptors: multi-functional therapeutic targets for sleeping disorders, eating disorders, drug addiction, cancers and other physiological disorders. Cell Signal. 2013;25(12):2413-2423. doi:10.1016/j.cellsig.2013.07.025

7. Dostert AH. Negative glucocorticoid receptor response elements and their role in glucocorticoid action. Curr Pharm Des. 2004;10:28072816.

8. Dallman MF, Akana SF, Pecoraro NC, et al. Glucocorticoids, the etiology of obesity and the metabolic syndrome. Curr Alzheimer Res. 2007;4:199-204.

9. Tomlinson JW, Stewart PM. Modulation of glucocorticoid action and the treatment of type-2 diabetes. Best Pract Res Clin Endocrinol Metab. 2007;21(4):607-619. doi:10.1016/j.beem.2007.07.003

10. DeFronzo RJ, Jequier E, Maeder E, Wahren J. The effect of insulin on the disposal of intravenous glucose: results from indirect calorimetry and hepatic and femoral venous catheterization. Diabetes. 1981;30:1000-1007. doi:10.2337/diab.30.12.1000

11. Richter EA, Hargreaves M. Exercise, GLUT4, and skeletal muscle glucose uptake. Physiol Rev. 2013;93:993-1017. doi:10.1152/physrev.00038.2012.-Glucose

12. Gerich JE. Is insulin resistance the principal cause of type 2 diabetes? Diabetes Obes Metab. 1999;1:257-263.

13. Hansen D, De Strijcker D, Calders P. Impact of endurance exercise training in the fasted state on muscle biochemistry and metabolism in healthy subjects: can these effects be of particular clinical benefit to type 2 diabetes mellitus and insulin-resistant patients? Sports Med. 2017;47(3):415-428. doi:10.1007/s40279-016-0594-x 
14. Pang B, Zhao LH, Zhou Q, et al. Application of berberine on treating type 2 diabetes mellitus. Int J Endocrinol. 2015;905749. doi:10.1155/ 2015/905749

15. Dong H, Wang N, Zhao L, et al. Berberine in the treatment of type 2 diabetes mellitus: a systemic review and meta-analysis. Evid Based Complement Alternat Med. 2012;591654. doi:10.1155/2012/591654

16. Dong H, Zhao Y, Zhao L, et al. The effects of berberine on blood lipids: a systemic review and meta-analysis of randomized controlled trials. Planta Med. 2013;79(6):437-446. doi:10.1055/s-0032-1328 321

17. Lee YS, Kim WS, Kim KH, et al. Berberine, a natural plant product, activates AMP-activated protein kinase with beneficial metabolic effects in diabetic and insulin-resistant states. Diabetes. 2006;55 (8):2256-2264. doi:10.2337/db06-0006

18. Tang LQ, Wei W, Chen LM, et al. Effects of berberine on diabetes induced by alloxan and a high-fat/high-cholesterol diet in rats. $J$ Ethnopharmacol. 2006;108(1):109-115. doi:10.1016/j.jep.2006.04. 019

19. Wang FL, Tang LQ, Yang F, et al. Renoprotective effects of berberine and its possible molecular mechanisms in combination of high-fat diet and low-dose streptozotocin-induced diabetic rats. Mol Biol Rep. 2013;40(3):2405-2418. doi:10.1007/s11033-012-2321-5

20. Zhang J, Yang JQ, He BC, et al. Berberine and total base from rhizoma coptis chinensis attenuate brain injury in an aluminuminduced rat model of neurodegenerative disease. Saudi Med J. 2009;30(6):760-766

21. Hou B, Zhao Y, Qiang G, et al. Puerarin mitigates diabetic hepatic steatosis and fibrosis by inhibiting TGF-beta signaling pathway activation in type 2 diabetic rats. Oxid Med Cell Longev. 2018;4545321. doi: $10.1155 / 2018 / 4545321$

22. Wei X, Tao J, Shen Y, et al. Sanhuang Xiexin Tang ameliorates type 2 diabetic rats via modulation of the metabolic profiles and NFkappaB/PI-3K/Akt signaling pathways. Front Pharmacol. 2018;9:955. doi:10.3389/fphar.2018.00955

23. Wang H, Zhu C, Ying Y, et al. Metformin and berberine, two versatile drugs in treatment of common metabolic diseases. Oncotarget. 2017;9(11):10135-10146.

24. Yin J, Xing H, Ye J. Efficacy of berberine in patients with type 2 diabetes mellitus. Metabolism. 2008;57(5):712-717. doi:10.1016/j. metabol.2008.01.013
25. Yue SJ, Liu J, Wang AT. Berberine alleviates insulin resistance by reducing peripheral branched-chain amino acids. Am J Physiol Endocrinol Metab. 2019;316(1):E73-E85. doi:10.1152/ajpendo.00256.2018

26. Zhang W, Xu YC, Guo FJ, et al. Anti-diabetic effects of cinnamaldehyde and berberine and their impacts on retinol-binding protein 4 expression in rats with type 2 diabetes mellitus. Chin Med J (Engl). 2008;121(21):2124-2128.

27. Petersen KF, Shulman GI. New insights into the pathogenesis of insulin resistance in humans using magnetic resonance spectroscopy. Obesity (Silver Spring). 2006;14 Suppl 1:34S-40S. doi:10.1038/ oby. 2006.280

28. Bruehl H, Rueger M, Dziobek I, et al. Hypothalamic-pituitary-adrenal axis dysregulation and memory impairments in type 2 diabetes. $J$ Clin Endocrinol Metab. 2007;92(7):2439-2445. doi:10.1210/jc.20062540

29. Levitt NS, Lambert E, Woods D, et al. Impaired glucose tolerance and elevated blood pressure in low birth weight, nonobese, young South African adults: early programming of cortisol axis. J Clin Endocrinol Metab. 2000;85:4611-4618.

30. Spencer RL, Deak T. A users guide to HPA axis research. Physiol Behav. 2017;178:43-65. doi:10.1016/j.physbeh.2016.11.014

31. Masuzaki H, Paterson J, Shinyama H, et al. A transgenic model of visceral obesity and the metabolic syndrome. Science. 2001;294 (5549):2166-2170. doi:10.1126/science.1066285

32. Dimitriadis G, Leighton B, Parry-Billings M, et al. Effects of glucocorticoid excess on the sensitivity of glucose transport and metabolism to insulin in rat skeletal muscle. Biochem J. 1997;321:707-712.

33. Piroli GG, Grillo CA, Reznikov LR, et al. Corticosterone impairs insulin-stimulated translocation of GLUT4 in the rat hippocampus. Neuroendocrinology. 2007;85(2):71-80. doi:10.1159/000101694

34. Ariga M, Nedachi T, Katagiri H, et al. Functional role of sortilin in myogenesis and development of insulin-responsive glucose transport system in C2C12 myocytes. J Biol Chem. 2008;283(15):1020810220. doi:10.1074/jbc.M710604200

35. Watson RT, Pessin JE. Intracellular organization of insulin signaling and GLUT4 translocation. Recent Prog Horm Res. 2001;56:175-193.

36. Yang J, Zhao P, Wan D, et al. Antidiabetic effect of methanolic extract from berberis julianae schneid. via activation of AMP-activated protein kinase in type 2 diabetic mice. Evid Based Complement Alternat Med. 2014;106206. doi:10.1155/2014/106206
Diabetes, Metabolic Syndrome and Obesity: Targets and Therapy is an international, peer-reviewed open-access journal committed to the rapid publication of the latest laboratory and clinical findings in the fields of diabetes, metabolic syndrome and obesity research. Original research, review, case reports, hypothesis formation, expert opinion and commentaries are all considered for publication. The manuscript management system is completely online and includes a very quick and fair peer-review system, which is all easy to use. Visit http://www.dovepress.com/testimonials.php to read real quotes from published authors. 